\title{
Pacto mundial: migrantes mexicanos frente al espejo de la economía estadounidense
}

\author{
The global compact: a view of Mexican \\ migrants within the us economy
}

Raúl Delgado Wise*
Selene Gaspar Olvera**

\begin{abstract}
Resumen. En septiembre de 2016 inició un proceso de consulta y negociación intergubernamental al interior de la ONU que culminará con la adopción de un pacto mundial en materia migratoria en 2018. El propósito de este trabajo es contribuir a desmitificar las concepciones dominantes del fenómeno migratorio, las cuales tienden a moldear la opinión pública e inciden en las negociaciones intergubernamentales alusivas al pacto mundial. Ante ese desafío y dada la importancia y el papel estratégico de la migración mexicana para la economía de Estados Unidos, se aportan indicadores y evidencia empírica que demuestra que lejos de constituir una carga para la economía receptora e independientemente de su estatus migratorio, los migrantes mexicanos y sus descendientes, coadyuvan, entre otras cosas, a satisfacer necesidades crecientes del mercado laboral, a impulsar el crecimiento económico y a fortalecer la seguridad social de la primera potencia capitalista y principal país receptor de migrantes del mundo.
\end{abstract}

Palabras clave. Pacto mundial, migración México-Estados Unidos, migración forzada, contribución económica de los inmigrantes, percepción de los migrantes.

\begin{abstract}
Within the UN, in September 2016, a process of intergovernmental consultation and negotiation was initiated that will culminate in the adoption of a Global compact on migration in 2018. The purpose of this paper is to contribute to demystify the dominant perceptions of the migratory phenomenon, shaping public opinion and influencing intergovernmental negotiations in the face of the Global compact. Faced with this challenge and given the importance and strategic role of Mexican migration for the United States economy, a series of indicators and empirical evidence are provided that demonstrate that, regardless of their migratory status, Mexican migrants including their descendants far from constituting a burden on the host economy, contribute, among other things, to meeting growing labor market needs, boosting economic growth, and strengthening social security of the first capitalist power and principal receiving country of migrants in the world.
\end{abstract}

Keywords. Global compact, Mexico-US migration, forced migration, economic contribution of immigrants, perception of migrants.

\footnotetext{
* Director de la Unidad Académica de Estudios del Desarrollo de la Universidad Autónoma de Zacatecas, presidente de la Red Internacional de Migración y Desarrollo y coordinador de la Cátedra UNESCO sobre Migración, Desarrollo y Derechos Humanos.

** Actuaria y maestra en Demografía Social por la Universidad Nacional Autónoma de México; investigadora de la Unidad Académica de Estudios del Desarrollo de la Universidad Autónoma de Zacatecas.
} 



\section{Introducción}

La discusión del tema migratorio al seno de la Organización de las Naciones Unidas (ONU), es relativamente reciente. Sus primeros antecedentes datan de la adopción de la Convención Internacional sobre la Protección de los Derechos Humanos de todos los Trabajadores Migratorios y de sus Familiares en 1990, la cual no ha sido ratificada aún por la mayoría de los países receptores de migrantes. A esa iniciativa le siguieron el Diálogo de Alto Nivel sobre Migración y Desarrollo (DAN), del cual se han celebrado a la fecha dos ediciones (en 2006 y 2013) en la sede central de la ONU; y el Foro Mundial de Migración y Desarrollo (FMMD), iniciativa derivada del DAN y organizada por los Estados miembros. Hasta ahora se han realizado nueve ediciones del FMMD: Bélgica (2007), Filipinas (2008), Grecia (2009), México (2010), Suiza (2011), Islas Mauricio (2012), Suecia (2014), Turquía (2015), Bangladesh (2016) y Berlín/Marruecos (2017/2018).

Ante los resultados relativamente limitados de las diferentes ediciones del DAN y los FMMD, el 19 de septiembre de 2016 jefes de Estado y de gobierno se reunieron en el marco de la Asamblea General de la ONU para debatir cuestiones relacionadas con la migración y los refugiados. Ese significativo encuentro envió un importante mensaje político acerca de la trascendencia de las políticas migratorias y de refugio en la agenda internacional. Con la adopción de la Declaración de Nueva York para los Refugiados y los Migrantes, los 193 Estados miembros de la ONU reconocieron la necesidad de un enfoque integral de movilidad humana y una mayor cooperación mundial. De ahí parte un extenso proceso de consultas regionales con expertos y organizaciones de la sociedad civil y varias negociaciones intergubernamentales tendientes a suscribir un pacto mundial en materia migratoria en una asamblea prevista para julio de 2018.

Este trabajo pretende integrarse a ese proceso, con la aportación de una serie de indicadores e información empírica en torno a un aspecto fundamental y, por lo general, soslayado en las inminentes negociaciones: las cuantiosas contribuciones que los migrantes hacen a las economías de los países de destino. Nuestro análisis se centra en un caso paradigmático en esta perspectiva: la migración México-Estados Unidos, y se fundamenta en estimaciones propias basadas en estadísticas oficiales de ambos países.

En efecto, los mexicanos constituyen el núcleo mayoritario de inmigrantes laborales en Estados Unidos, que a su vez figura como la primera potencia 
económica y el país con más número de inmigrantes. También comprenden el segmento de la clase trabajadora residente que detenta la mayor proporción de indocumentados, circunstancia que los sitúa en condiciones de acendrada vulnerabilidad. De forma similar, experimentan cotidianamente situaciones de severa discriminación, exclusión social, precariedad laboral y explotación extrema.

Las evidencias resultan contundentes y destacan la necesidad de llevar a cabo una operación de desmitificación en varios planos para demostrar la participación de los mexicanos en la economía estadounidense en términos de demanda laboral, producción, servicios y seguridad social. Así, el artículo expone diversos indicadores y datos de 1994 a 2016, periodo de vigencia del Tratado de Libre Comercio de América del Norte (TLCAN), e incluye los impactos de la crisis desencadenada en 2007.

\section{Aporte laboral}

Desde la implementación del TLCAN los migrantes mexicanos han cumplido una función clave en la satisfacción de la demanda laboral estadounidense, pese a que casi la mitad carga con el estigma de «ilegales» y las consecuencias que se derivan en cuanto a explotación laboral y vulnerabilidad social. De los 30.4 millones de nuevos empleos creados en Estados Unidos entre 1994 y 2016, 13.4 por ciento fue ocupado por trabajadores nacidos en México. Más aún, ante la incapacidad de la población nativa para cubrir las necesidades laborales, 46.1 por ciento de la demanda fue satisfecha mediante el concurso de trabajadores inmigrantes, de los cuales poco más de 4 millones eran mexicanos. Cabe destacar la participación de la población nativa de origen mexicano en el crecimiento del empleo, su número se elevó a cerca de 5 millones, lo que representa 15.8 por ciento del crecimiento total. Tan requerida y apreciada ha sido la oferta de trabajadores mexicanos y de sus descendientes que su ocupación se incrementó en 121.3 y 140.4 por ciento respectivamente, frente a un crecimiento de 25.2 por ciento del empleo total en Estados Unidos (gráfica 1). ${ }^{1}$

${ }^{1}$ Para los fines de este estudio se clasifica a la población por lugar de nacimiento y origen étnico en los siguientes grupos poblacionales: 1) inmigrantes mexicanos: nacidos en México, 2) inmigrantes no mexicanos: nacidos en un país distinto a México o Estados Unidos, 3) nativos mexicoamericanos: 
GRÁFICA 1

Estados Unidos. Contribución porcentual

al incremento absoluto del empleo 1994-2016

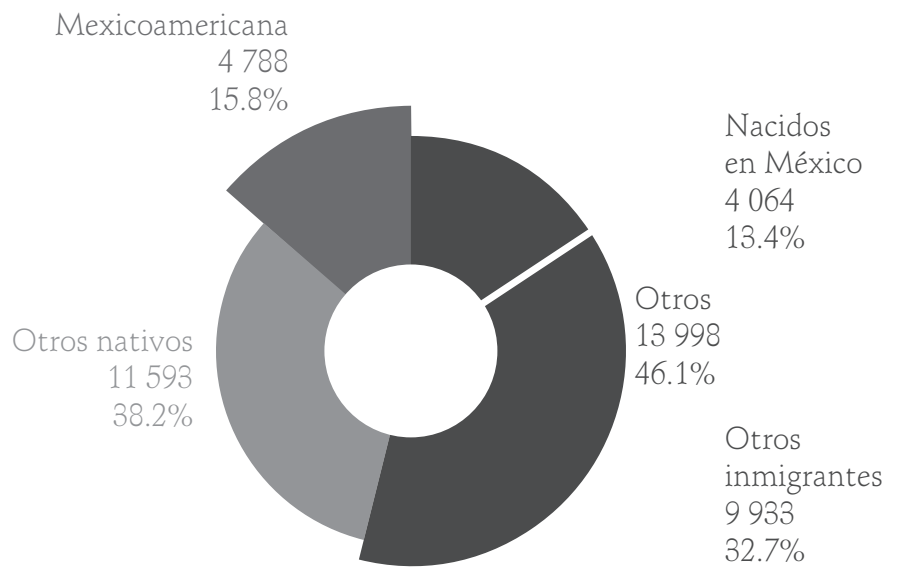

Fuente: estimación de los autores con base en U.S. Bureau of Labor Statistics, Current Population Survey (CPS), March supplement, 1994 y 2016.

La crisis económica de finales de 2007 afectó a todos los trabajadores sin distinción de origen nacional. Entre 2008 y 2016, el empleo global se elevó en 4.0 por ciento pese a la contracción económica de Estados Unidos y las variaciones en el crecimiento del empleo, los inmigrantes mexicanos ocuparon 7.1 por ciento de los 5.7 millones de nuevos empleos e incrementaron su participación en 5.8 por ciento, lo que en términos absolutos equivale a 406 mil nuevos empleos. Es pertinente recordar que si bien en 2009 la tasa de desempleo de los mexicanos en ese país alcanzó su máximo histórico con 13.3 por ciento (Delgado y Gaspar, 2012), su recuperación fue muy rápida y al siguiente año descendió a 12.6 por ciento y continuó disminuyendo hasta 5.1 por ciento en 2016, inferior incluso a la que tenía en 2007 (5.5 por ciento). Por su parte, 2.2 millones de nativos de origen mexicano se sumaron al mercado laboral en el mismo lapso, aumentó su número en 37 por ciento y ocuparon 38.5 por ciento del total de empleos creados (cuadro 1).

Es preciso aclarar que el movimiento de la migración mexicana a Estados Unidos se correlaciona positivamente con la tasa de desempleo en ese país; es

nacidos en Estados Unidos con uno o ambos padres nacidos en México o que se declaran de origen mexicano, 4) otros nativos: nacidos en Estados Unidos de origen no mexicano. 
decir, uno de los motores principales de la emigración mexicana ha sido la demanda de empleo. Referente a la contracción económica, la tasa de desempleo de los inmigrantes mexicanos aumenta y, ante el menor indicio de recuperación económica, desciende. Al respecto, es erróneo considerar que los mexicanos están quitando empleos a los nativos. En cualquier caso, como se abordará después, no se trata de un simple juego de oferta y demanda laboral, porque en el trasfondo de tal comportamiento subyace una estrategia corporativa tendiente a reducir costos laborales, sin importar sus repercusiones para los migrantes y sus familias ni para la clase trabajadora en general. De hecho, mientras la población nativa blanca de ascendencia no extranjera disminuyó año con año (0.4 por ciento anual entre 2008 y 2016) por un efecto del envejecimiento, la participación de la población nativa de origen extranjero en la fuerza laboral se incrementó a una tasa de 1.9 por ciento anual en el mismo periodo (Gaspar, 2017). Se prevé que el número de estadounidenses aumentará a más del doble, al pasar de 46 millones en 2016 a más de 98 millones en 2060 (Mather, Jacobsen y Pollard, 2015).

\section{CUADRO 1}

Crecimiento de empleo en Estados Unidos según grupo de origen étnico

\begin{tabular}{|c|c|c|c|c|c|c|c|c|c|c|}
\hline \multirow{3}{*}{$\begin{array}{l}\text { Población ocupada } \\
\text { (miles de personas) }\end{array}$} & \multirow{3}{*}{1994} & \multirow{3}{*}{2008} & \multirow{3}{*}{2016} & \multicolumn{3}{|c|}{ 1994-2008 } & \multicolumn{3}{|c|}{ 2008-2016 } & \multirow{3}{*}{ 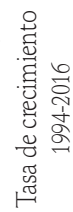 } \\
\hline & & & & \multicolumn{2}{|c|}{ Crecimiento } & \multirow{2}{*}{ 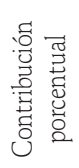 } & \multicolumn{2}{|c|}{ Crecimiento } & \multirow{2}{*}{ 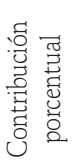 } & \\
\hline & & & & to & $\%$ & & Absoluto & $\%$ & & \\
\hline Población ocupada & 120383 & 145013 & (200 & पा & 20.5 & 100.0 & 5749 & 4.0 & 100.0 & 1.0 \\
\hline $\begin{array}{l}\text { Población ocupada } \\
\text { nativa }\end{array}$ & 107416 & 121405 & 123797 & 13988 & 13.0 & 56.8 & 2392 & 2.0 & 41.6 & \\
\hline Mexicoamericana & 3409 & $590 \mathrm{~J}$ & 8197 & J 10 & 7.0 & 10.5 & 2212 & 37.0 & 38.5 & 4.1 \\
\hline Otros nativos & 104007 & 115419 & 115600 & 11412 & 11.0 & 46.3 & 181 & 0.2 & 3.1 & 0.5 \\
\hline $\begin{array}{l}\text { Población ocupada } \\
\text { migrante }\end{array}$ & 12967 & 23608 & 26965 & 10641 & 82.1 & 43.2 & 3357 & 14.2 & 58.4 & \\
\hline Mexicana & 3350 & 7009 & 7415 & 3659 & 109.2 & 14.9 & 406 & 5.8 & 7.1 & 8.7 \\
\hline No mexicana & 9617 & 16599 & 19550 & 6982 & 72.6 & 28.3 & 2951 & 17.8 & 51.3 & 3.3 \\
\hline
\end{tabular}

Fuente: estimación de los autores con base en US Burearu of Labor Statistics, Current Population Survey (CPS), March supplement, 1994, 2008 y 2016.

El impacto de la migración mexicana hacia Estados Unidos ha sido contrastante para ambos países. En México ha sido el fruto de un proceso de integración asimétrico y subordinado que ha traído consigo el desmantelamiento de los 
aparatos productivo, comercial y de servicios, bajo una lógica de maquilización y extractivismo acompañada de una disminución en la capacidad para generar empleo con el consecuente desencadenamiento de dinámicas de expulsión y descomposición social (Cypher y Delgado, 2011). A tal grado se ha dado este proceso que el país ha tenido uno de los peores desempeños en crecimiento económico de toda América Latina. En contraste, en Estados Unidos el proceso ha contribuido a nutrir y flexibilizar la oferta laboral, abaratar costos e incrementar los beneficios al capital.

Acontece en la manufactura un doble movimiento observado desde 1994 y que prevalece hasta la fecha. Por un lado, se aprecia una sustitución de trabajadores nativos por migrantes (cuadro 2). Es necesario considerar que en los periodos 1994-2008 y 2008-2016, el personal nativo ocupado en ese sector disminuyó en 4.3 millones y casi 5.6 millones, respectivamente, al tiempo que los migrantes ocupados aumentaron en 411 mil y disminuyeron en 394 mil. Concerniente a los inmigrantes mexicanos, mantuvieron un crecimiento positivo: poco más de 163 mil trabajadores se sumaron entre 1994 y 2008 y 246 mil entre 2008 y 2016. Por otro lado, para ciertos grupos de inmigrantes, en particular los mexicanos, se otorgaron niveles salariales muy bajos (cuadro 2). En 2016, el salario promedio anual de un migrante mexicano ocupado en la manufactura equivalía al que percibía un nativo en ese sector en 1994. Mientras los trabajadores nativos de origen no mexicano ganaron en promedio casi 55 mil dólares al año (25.5 por hora) en 2016, los inmigrantes mexicanos percibieron menos de 32 mil dólares (15.6 dólares por hora) y los nativos de origen mexicano 40 mil dólares (19.3 dólares por hora). De acuerdo con Scott (2015), las principales causas de la pérdida de empleo en la industria manufacturera, que comenzó en 1998, obedecieron al creciente déficit comercial con China, México y otras naciones de bajos salarios, así como a las recesiones de 2001 y 2007. Se trata, en pocas palabras, del imperio del arbitraje laboral global como pivote de la estrategia de dominación imperialista comandada por las grandes corporaciones multinacionales de base estadounidense (Suwandi y Foster, 2016).

Desde el inicio de la recesión económica en 2007 hasta marzo de 2011 se perdieron poco más de 7.4 millones de empleos en Estados Unidos. Si bien 6 por ciento de los inmigrantes mexicanos fue afectado, los nativos de origen mexicano incrementaron un poco su participación en el empleo y debido a sus relativamente bajos ingresos amortiguaron hasta cierto punto el impacto negativo de la crisis sobre la economía estadounidense (Gaspar, 2017). 


\section{CUADRO 2}

Ocupados en la manufactura según condición migratoria 1994, 2008 y 2016

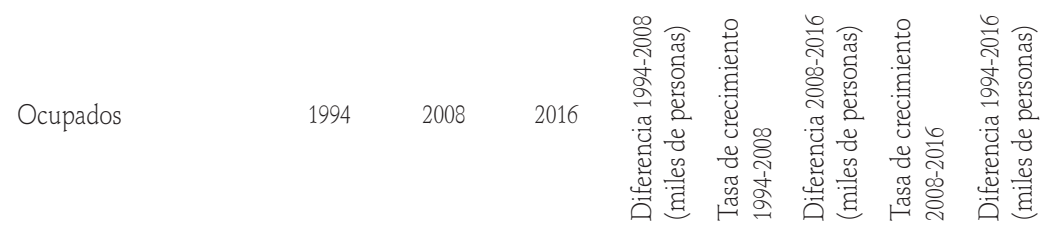

\begin{tabular}{|c|c|c|c|c|c|c|c|c|}
\hline \multicolumn{9}{|l|}{ Manufactura } \\
\hline $\begin{array}{l}\text { Población ocupada } \\
\text { (miles de personas) }\end{array}$ & 19758 & 15843 & 9891 & \multirow[t]{2}{*}{-3915} & \multirow[t]{2}{*}{-1.6} & \multirow[t]{2}{*}{-5952} & \multirow[t]{2}{*}{-5.7} & \multirow[t]{2}{*}{-9867} \\
\hline Salario promedio anual (dólares) & 29013 & 47316 & 52626 & & & & & \\
\hline Población ocupada nativa & 17180 & 12853 & 7296 & \multirow[t]{2}{*}{-4327} & \multirow[t]{2}{*}{-2.1} & \multirow[t]{2}{*}{-5557} & \multirow[t]{2}{*}{-6.8} & \multirow[t]{2}{*}{-9884} \\
\hline Salario promedio anual (dólares) & 29688 & 48637 & 54018 & & & & & \\
\hline Mexicoamericana & 463 & 593 & 577 & \multirow[t]{2}{*}{130} & \multirow[t]{2}{*}{1.8} & \multirow[t]{2}{*}{-16} & \multirow[t]{2}{*}{-0.3} & \multirow[t]{2}{*}{114} \\
\hline Salario promedio anual (dólares) & 24757 & 35335 & 40499 & & & & & \\
\hline Resto de nativos & 16717 & 12260 & 6719 & \multirow[t]{2}{*}{-4456} & \multirow[t]{2}{*}{-2.2} & \multirow[t]{2}{*}{-5542} & \multirow[t]{2}{*}{-7.2} & \multirow[t]{2}{*}{-9998} \\
\hline Salario promedio anual (dólares) & 29824 & 49279 & 54838 & & & & & \\
\hline Población ocupada migrante & 2578 & 2990 & 2595 & \multirow[t]{2}{*}{411} & \multirow[t]{2}{*}{1.1} & \multirow[t]{2}{*}{-394} & \multirow[t]{2}{*}{-1.8} & 17 \\
\hline Salario promedio anual (dólares) & 24389 & 41555 & 42351 & & & & & \\
\hline Mexicana & 904 & 1067 & 1314 & \multirow[t]{2}{*}{163} & \multirow[t]{2}{*}{1.2} & \multirow[t]{2}{*}{246} & \multirow[t]{2}{*}{2.6} & \multirow[t]{2}{*}{409} \\
\hline Salario promedio anual (dólares) & 15691 & 27190 & 31996 & & & & & \\
\hline No mexicana & 1674 & 1922 & 1282 & \multirow[t]{2}{*}{248} & \multirow[t]{2}{*}{1.0} & \multirow[t]{2}{*}{-640} & \multirow[t]{2}{*}{-4.9} & -392 \\
\hline Salario promedio anual (dólares) & 29093 & 49655 & 54354 & & & & & \\
\hline & & & & & 1994 & 2008 & & 2016 \\
\hline Diferencia salarial del nativo versus & rante $m$ & cano & & & 13997 & 21447 & & 2022 \\
\hline Diferencia salarial del migrante no & icano ve & $s$ mexica & & & 13402 & 22465 & & 2359 \\
\hline $\begin{array}{l}\text { \% de la población ocupada nativa } \\
\text { manufactura }\end{array}$ & to al to & de ocup & sen & & 87.0 & 81.1 & & 73.8 \\
\hline $\begin{array}{l}\% \text { de población ocupada migrante } \\
\text { ocupados en manufactura }\end{array}$ & xicana & ecto a & al de & & 8.5 & 8.5 & & 8.5 \\
\hline $\begin{array}{l}\text { \% de población ocupada migrante } \\
\text { en manufactura }\end{array}$ & cana res & cto al tot & de ocupa & dos & 4.6 & 6.7 & & 13.3 \\
\hline $\begin{array}{l}\text { \% de población ocupada mexicoam } \\
\text { en manufactura }\end{array}$ & na resp & al tot & ocupad & & 2.3 & 3.7 & & 5.8 \\
\hline
\end{tabular}

Fuente: estimación de los autores con base en US Bureau of Labor Statistics, Current Population Survey (CPS), March supplement, 1994, 2008 y 2016.

Los sectores económicos más perjudicados entre la población de origen mexicano (inmigrante y nativa) entre 2008 y 2016 fueron la construcción y la manufactura, 
en ese orden de importancia. En cambio, se registraron aumentos en los sectores de administración pública, minería y agricultura, silvicultura, pesca y caza (cuadro 3). Asimismo, aunque en menor medida, la cifra de trabajadores de origen mexicano ocupados en administración pública creció en 9.2 por ciento (30 mil 444 nuevos empleos). Los sectores de mayor crecimiento para la población nativa de origen mexicano fueron agricultura, silvicultura, pesca y caza (92.0 por ciento), recreativos y hospitalidad (84.7 por ciento), minería (64.5 por ciento), servicios profesionales y empresariales (56.3 por ciento), transporte y servicios públicos (51.9 por ciento) y educación y servicios de salud (42.9 por ciento).

\section{CUADRO 3}

Importancia relativa de la población de origen mexicano (inmigrante y nativa) en 2016 y crecimiento del empleo entre 2008 y 2016

\begin{tabular}{|c|c|c|c|c|c|c|c|}
\hline & 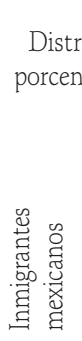 & 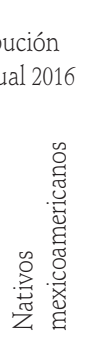 & 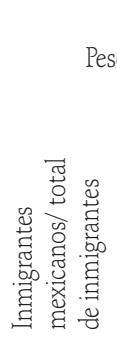 & 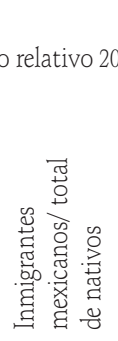 & 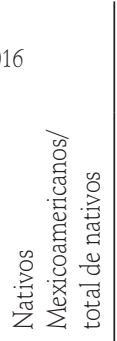 & 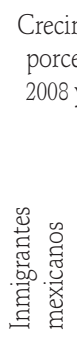 & 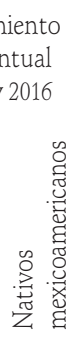 \\
\hline Agricultura, silvicultura, pesca y caza & 6.4 & 0.9 & 80.1 & $24-8$ & 3.8 & 31.5 & 92.0 \\
\hline Minería & 0.6 & 0.9 & 38.1 & 6.6 & 9.9 & 48.2 & 64.5 \\
\hline Construcción & 17.7 & 7.0 & 50.6 & 18.0 & 7.9 & -12.8 & 18.8 \\
\hline Manufactura & 12.9 & 8.3 & 32.0 & 7.9 & 5.7 & -10.1 & 15.3 \\
\hline Comercio al mayoreo y menudeo & 10.4 & 16.2 & 24.0 & 4.6 & 7.8 & 0.2 & 28.6 \\
\hline Transporte y servicios públicos & 4.0 & 6.7 & 20.8 & 4.5 & 8.3 & 18.3 & 51.9 \\
\hline Información & 0.6 & 1.5 & 11.4 & 1.7 & 4.8 & 6.8 & 2.4 \\
\hline Actividades financieras & 2.7 & 5.9 & 14.1 & 2.2 & 5.4 & 29.7 & 19.4 \\
\hline Servicios profesionales y empresariales & 13.0 & 10.2 & 25.1 & 6.8 & 5.9 & 25.1 & 56.3 \\
\hline Educación y servicios de salud & 9.0 & 21.4 & 13.3 & 2.2 & 5.8 & 24.5 & 42.9 \\
\hline Recreativos y hospitalidad & 15.3 & 12.3 & 36.5 & 10.3 & 9.2 & 8.3 & 84.7 \\
\hline Otros servicios & 5.8 & 4.3 & 28.3 & 8.0 & 6.5 & 5.0 & 30.3 \\
\hline Administración pública & 1.4 & 4.4 & 15.6 & 1.7 & 5.9 & 84.2 & 9.2 \\
\hline
\end{tabular}

Fuente: estimación de los autores con base en US Bureau of Labor Statistics, Current Population Survey (CPS), March supplement, 2008 y 2016. 
La precariedad del mercado laboral se agudiza con el reemplazo de trabajadores, la inestabilidad o la fragilidad en el empleo y el marcado declive en los estándares laborales en un contexto de desmantelamiento sindical. Este proceso forma parte de la estrategia de desindustrialización de la economía estadounidense y la configuración de redes globales de capital, donde el modelo mexicano de exportación de fuerza de trabajo barata desempeña un papel central.

\section{Contribución a la producción}

La gran cantidad de migrantes mexicanos que residen y trabajan en Estados Unidos participa de manera notable en el crecimiento económico de la economía más poderosa del mundo. Su contribución al producto interno bruto (PIB)se elevó entre 1998 y 2016 en 172 por ciento, mientras que el PIB nacional creció en 41.8 por ciento anual. ${ }^{2}$ En 2016 tal aporte ascendió a 819 mil millones de dólares (mmdd), cifra que corresponde a 4.9 por ciento del PIB estadounidense y 66 por ciento del PIB mexicano. Complementariamente, la población nativa de origen mexicano colaboró con 10.6 por ciento, lo que en términos absolutos equivale a un incremento de 547 mmdd entre 1998 y 2016. En conjunto, la población de origen mexicano (inmigrante y nativa) contribuyó con 19.4 por ciento (gráficas 2 y 3).

Resulta pertinente subrayar el hecho de que entre 2008 y 2016, pese a la crisis, el PIB de Estados Unidos creció en términos reales 1832 mmdd (a precios de 2009). A dicho aumento los mexicanos aportaron 103 mmdd, lo que corresponde a 5.6 por ciento. El PIB de la población nativa de origen mexicano creció en 16.0 por ciento con 294 mmdd, lo que en conjunto (migrantes mexicanos y sus descendientes) implicó un incremento de 21.6 por ciento. Entre 2008 y 2016, la contribución de los inmigrantes al crecimiento del PIB estadounidense fue de 15.9 por ciento; el grupo de inmigrantes que más aportó fue el de los mexicanos con 35.2 por ciento (véase cuadro 4).

${ }^{2}$ La estimación de la participación del PIB se basa en la metodología propuesta por Alejandro Canales (2010) como parte de los trabajos de Simde. Fue realizada con datos de Current population Survey (CPS), suplemento de marzo 1998-2016, e información del Buró de Análisis Económico (BEA) 1998-2016 base 2009. 


\section{GRÁFICA 2}

Contribución de los migrantes mexicanos y mexicoamericanos al PIB 1996-2016

PIB mexicanos en Estados Unidos

PIB de los nativos de origen mexicano

- PIB de la población de origen mexicano / PIB Estados Unidos

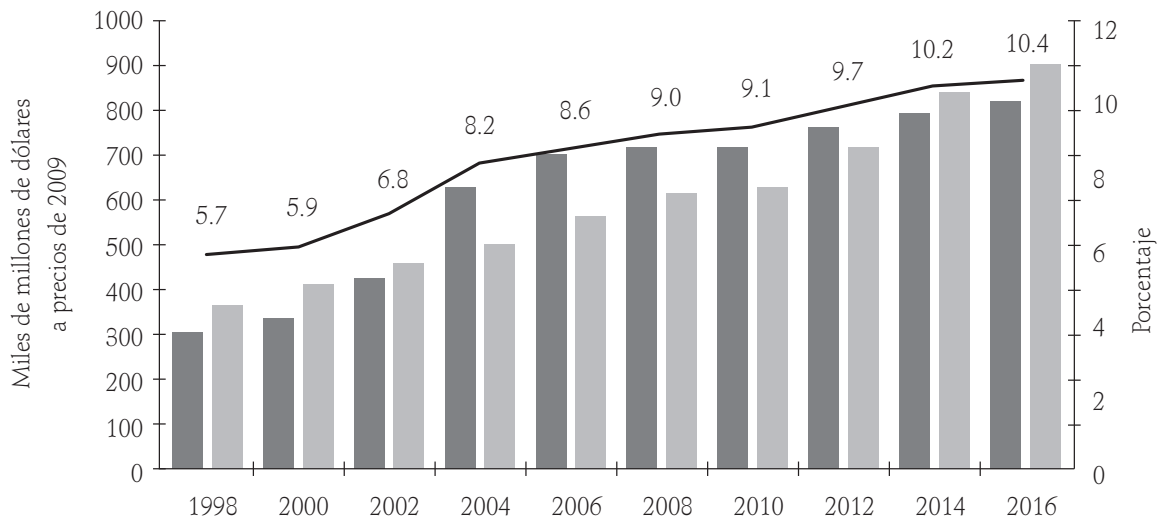

Fuente: estimaciones de los autores basadas en U.S. Bureau of Economic Analysis, Gross Domestic Product by Industry Accounts, 1998 a 2016, y Current Population Survey (CPS), March supplement, 1998-2016.

GRÁFICA 3

Contribución al crecimiento del PIB en Estados Unidos por origen 1998 y 2016

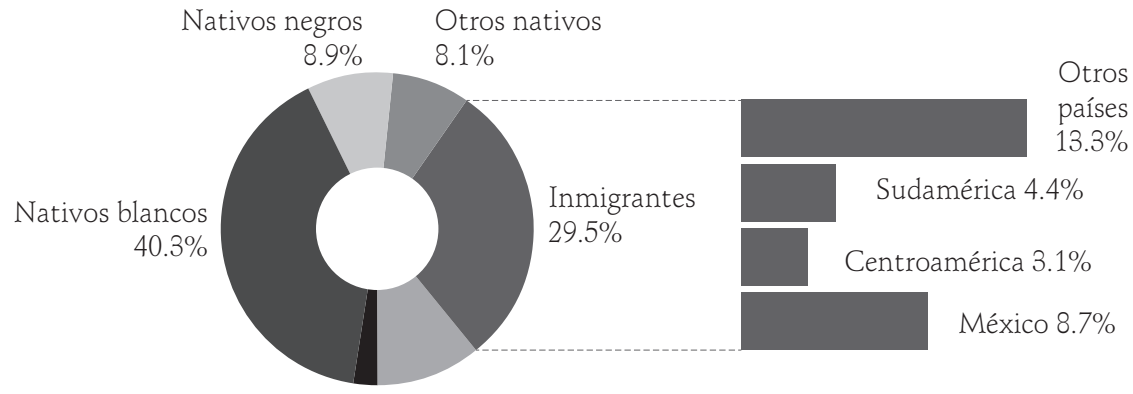

Latinoamericanos de Mexicanos de segunda segunda y tercera generación y tercera generación $2.5 \% \quad 10.7 \%$

Fuente: estimaciones de los autores basadas en U.S. Bureau of Economic Analysis, Gross Domestic Product by Industry Accounts, 1998 a 2016, y Current Population Survey (CPS), March supplement, 1998-2016. 


\section{CUADRO 4}

Indicadores del PIB y fuerza de trabajo 2007-2016

PIB (mmdd a precios de 2009)

\begin{tabular}{|c|c|c|c|c|c|c|c|c|c|}
\hline \multirow[b]{2}{*}{ Año } & \multicolumn{4}{|c|}{ PIB (mmdd a precios de 2009) } & \multicolumn{2}{|c|}{$\begin{array}{l}\text { Importancia del } \\
\text { aporte de los } \\
\text { mexicanos al PIB }\end{array}$} & \multirow{2}{*}{ 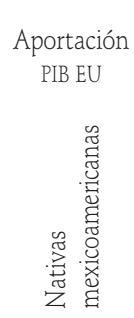 } & \multicolumn{2}{|c|}{$\begin{array}{c}\text { Aporte a la fuerza } \\
\text { laboral }\end{array}$} \\
\hline & 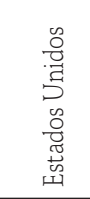 & 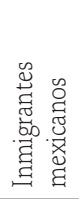 & 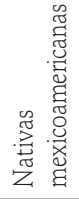 & 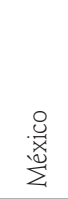 & 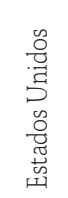 & 荧 & & 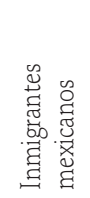 & 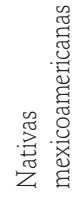 \\
\hline 2007 & 14874 & 741 & 582 & 1035 & $5.0 \%$ & $71.6 \%$ & $3.9 \%$ & $5.0 \%$ & $3.9 \%$ \\
\hline 2008 & 14830 & 717 & 612 & 1049 & $4.8 \%$ & $68.3 \%$ & $4.1 \%$ & $4.8 \%$ & $4.1 \%$ \\
\hline 2009 & 14419 & 690 & 620 & 1000 & $4.8 \%$ & $69.0 \%$ & $4.3 \%$ & $4.8 \%$ & $4.3 \%$ \\
\hline 2010 & 14784 & 726 & 624 & 1051 & $4.9 \%$ & $69.0 \%$ & $4.2 \%$ & $4.9 \%$ & $4.2 \%$ \\
\hline 2011 & 15021 & 723 & 653 & 1094 & $4.8 \%$ & $66.1 \%$ & $4.3 \%$ & $4.8 \%$ & $4.3 \%$ \\
\hline 2012 & 15355 & 763 & 721 & 1138 & $5.0 \%$ & $67.1 \%$ & $4.7 \%$ & $5.0 \%$ & $4.7 \%$ \\
\hline 2013 & 15612 & 763 & 721 & 1153 & $4.9 \%$ & $66.2 \%$ & $4.9 \%$ & $4.9 \%$ & $4.9 \%$ \\
\hline 2014 & 15982 & 793 & 842 & 1179 & $5.0 \%$ & $67.3 \%$ & $5.3 \%$ & $5.0 \%$ & $5.3 \%$ \\
\hline 2015 & 16397 & 842 & 870 & 1210 & $5.1 \%$ & $69.6 \%$ & $5.3 \%$ & $5.1 \%$ & $5.3 \%$ \\
\hline 2016 & 16662 & 819 & 906 & 1238 & $4.9 \%$ & $66.2 \%$ & $5.4 \%$ & $4.9 \%$ & $5.4 \%$ \\
\hline 008-2016 & 1832 & 103 & 294 & & $5.6 \%$ & & $16.0 \%$ & $7.1 \%$ & $38.5 \%$ \\
\hline
\end{tabular}

Fuente: estimación de los autores con base en US Bureau of Labor Statistics, Current Population Survey (CPS), March supplement, 2003-2016, BEA, Banco Mundial. El ejercicio se realizó aplicando la metodología propuesta por Simde.

\section{Subsidio a la seguridad social}

Con frecuencia suele afirmarse que los migrantes constituyen una carga fiscal y social para las naciones receptoras. Se trata de otro gran mito que contraviene la evidencia. Los migrantes mexicanos residentes en Estados Unidos aportaron al fondo fiscal $14.5 \mathrm{mmdd}$ por concepto de impuestos directos al trabajo y de impuestos indirectos vía consumo (44.3 mmdd) en 2008 (cuadros $5 a$ y 5b). Esa cantidad duplica el monto total de remesas enviado a México e irónicamente equivale al presupuesto de la Patrulla Fronteriza, el cual en el mismo año fue de 53 mmdd, según el Department of Homeland Security.

En la estadística del cuadro 5 a se advierte que el pago de impuesto al trabajo de los mexicanos (26 mmdd en 2016) representa casi una aportación neta al fisco de Estados Unidos, pues los ingresos percibidos por concepto de asistencia infantil, 
educación, asistencia pública, seguridad social, desempleo, ingreso para veteranos y discapacidad, sólo ascienden a 309 mmdd (2.4 por ciento de las aportaciones totales). Tales ingresos son inferiores a los recibidos por el resto de los grupos poblacionales. En general se observa que la población inmigrante mexicana y su descendencia dieron mucho más de lo que recibieron en pago de impuestos y de los servicios públicos recibidos (cuadros 5 a y $5 b$ ).

\section{CUADRO 5A}

Pago de impuestos sobre el producto al salario e ingresos por fuente de la población ocupada según grupo de origen en Estados Unidos (varios años)

\begin{tabular}{|c|c|c|c|c|c|c|}
\hline Millones de dólares & 1994 & 2000 & 2005 & 2008 & 2010 & 2016 \\
\hline \multirow[t]{2}{*}{ Total de impuesto por trabajo } & 411741 & 608910 & 759698 & 873476 & 860960 & 1067118 \\
\hline & \multicolumn{6}{|c|}{ Migrantes mexicanos } \\
\hline Pago de impuesto & 2686 & 5827 & 11742 & 14468 & 13409 & 26029 \\
\hline \% de participación en el impuesto total & 0.7 & 1.0 & 1.5 & 1.7 & 1.6 & 2.4 \\
\hline Ingresos por tipo de fuente & 108 & 94 & 164 & 159 & 291 & 309 \\
\hline Ingresos por asistencia infantil & 6 & 15 & 31 & 34 & 47 & 54 \\
\hline Ingresos por asistencia educativa & 8 & 6 & 28 & 24 & 29 & 56 \\
\hline Asistencia pública & 14 & 7 & 9 & 14 & 6 & 13 \\
\hline Seguridad social & 32 & 30 & 45 & 42 & 77 & 120 \\
\hline Ingresos por desempleo & 45 & 34 & 48 & 43 & 124 & 57 \\
\hline Ingresos veteranos & 3 & 2 & 1 & 0 & 8 & 10 \\
\hline Ingreso discapacidad & 0 & 0 & 0 & 1 & 0 & 0 \\
\hline \multirow[t]{2}{*}{ Total ingreso/pago impuesto } & 4.0 & 1.6 & 1.4 & 1.1 & 2.2 & 2.4 \\
\hline & \multicolumn{6}{|c|}{ Migrante no mexicano } \\
\hline Pago impuesto & 27227 & 49909 & 76524 & 95139 & 92176 & 139157 \\
\hline \% de participación en el impuesto total & 6.6 & 8.2 & 10.1 & 10.9 & 10.7 & 13.0 \\
\hline Ingreso por tipo de fuente & 710 & 900 & 900 & 1008 & 1298 & 1767 \\
\hline Ingreso por asistencia infantil & 108 & 123 & 123 & 143 & 117 & 152 \\
\hline Ingreso por asistencia educativa & 161 & 219 & 219 & 288 & 237 & 490 \\
\hline Asistencia pública & 32 & 17 & 17 & 11 & 12 & 26 \\
\hline Seguridad social & 285 & 374 & 374 & 465 & 597 & 902 \\
\hline Ingresos por desempleo & 78 & 127 & 127 & 69 & 289 & 95 \\
\hline Ingresos veteranos & 3 & 2 & 1 & 0 & 8 & 10 \\
\hline Ingreso discapacidad & 5 & 0 & 0 & 0 & 0 & 5 \\
\hline Total ingresos/pago impuesto & 2.6 & 1.8 & 1.2 & 1.1 & 1.4 & 2.5 \\
\hline
\end{tabular}

Fuente: estimación de los autores con base en US Bureau of Labor Statistics, Current Population Survey (CPS), March supplement, 1994, 2000, 2005, 2008, 2010 y 2016 y Federal Insurance Contributions Act. Se aplicó la metodología propuesta por Héctor Rodríguez como parte de los trabajos del Simde. 


\section{CUADRO 5B}

Pago de impuestos sobre el producto al salario e ingresos por fuente de la población ocupada según grupo de origen en Estados Unidos (varios años)

\begin{tabular}{|c|c|c|c|c|c|c|}
\hline Millones de dólares & 1994 & 2000 & 2005 & 2008 & 2010 & 2016 \\
\hline \multirow[t]{2}{*}{ Total de impuesto por trabajo } & 411741 & 608910 & 759698 & 873476 & 860960 & 1067118 \\
\hline & \multicolumn{6}{|c|}{ Nativos mexicoamericanos } \\
\hline Pago de impuesto & 4638 & 8251 & 12704 & 14919 & 15641 & 30360 \\
\hline $\begin{array}{l}\% \text { de participación en el impuesto } \\
\text { total }\end{array}$ & 1.1 & 1.4 & 1.7 & 1.7 & 1.8 & 2.8 \\
\hline Ingresos por tipo de fuente & 172 & 273 & 367 & 390 & 527 & 690 \\
\hline Ingresos por asistencia infantil & 28 & 48 & 75 & 84 & 67 & 98 \\
\hline Ingresos por asistencia educativa & 34 & 59 & 105 & 90 & 129 & 247 \\
\hline Asistencia pública & 21 & 27 & 10 & 18 & 11 & 16 \\
\hline Seguridad social & 42 & 88 & 97 & 130 & 136 & 161 \\
\hline Ingresos por desempleo & 42 & 40 & 52 & 46 & 150 & 83 \\
\hline Ingresos veteranos & 4 & 10 & 27 & 22 & 34 & 80 \\
\hline Ingreso discapacidad & 0 & 0 & 0 & 0 & 0 & 5 \\
\hline \multirow[t]{2}{*}{ Total ingreso/pago impuesto } & 3.7 & 3.3 & 2.9 & 2.6 & 3.4 & 4.5 \\
\hline & \multicolumn{6}{|c|}{ Otros nativos } \\
\hline Pago impuesto & 377190 & 544923 & 658727 & 748950 & 739653 & 871572 \\
\hline $\begin{array}{l}\% \text { de participación en el impuesto } \\
\text { total }\end{array}$ & 91.6 & 89.5 & 86.7 & 85.7 & 85.9 & 81.7 \\
\hline Ingreso por tipo de fuente & 7100 & 8697 & 11431 & 12018 & 14984 & 16936 \\
\hline Ingreso por asistencia infantil & 1089 & 1530 & 1827 & 1660 & 1494 & 1492 \\
\hline Ingreso por asistencia educativa & 1211 & 1695 & 2152 & 2218 & 2360 & 3222 \\
\hline Asistencia pública & 283 & 201 & 98 & 95 & 101 & 113 \\
\hline Seguridad social & 2812 & 3925 & 5488 & 6130 & 7094 & 9662 \\
\hline Ingresos por desempleo & 1286 & 738 & 1171 & 1073 & 2970 & 954 \\
\hline Ingresos veteranos & 408 & 577 & 685 & 809 & 959 & 1424 \\
\hline Ingreso discapacidad & 11 & 31 & 10 & 34 & 6 & 68 \\
\hline Total ingresos/pago impuesto & 1.9 & 1.6 & 1.7 & 1.6 & 2.0 & 3.9 \\
\hline
\end{tabular}

Fuente: estimación de los autores con base en US Bureau of Labor Statistics, Current Population Survey (CPS), March supplement, 1994, 2000, 2005, 2008, 2010 y 2016 y Federal Insurance Contributions Act. Se aplicó la metodología propuesta por Héctor Rodríguez como parte de los trabajos del Simde.

Los migrantes mexicanos ocupados carecen de una amplia gama de servicios sociales, tampoco tienen acceso a la seguridad social ni a programas de asistencia pública; la mayoría de los asalariados ocupa el rubro más bajo en la percepción de ingresos y presenta los índices de pobreza más altos. De acuerdo 
con la Encuesta Continua de Población 2016 (CPS, por sus siglas en inglés), 2.7 millones de migrantes mexicanos residentes en Estados Unidos se ubicaron en la categoría de pobres y entre los mexicanos ocupados poco más de un millón se encuentra en esa situación; en tanto, en 2011 esas cifras eran de 3.5 y 1.4 millones respectivamente. Su descendencia observa proporciones similares de pobres, 22.4 por ciento de los 24.8 millones de nativos de ascendencia mexicana (5.6 millones). En adición, los niveles educativos de los mexicanos siguen siendo relativamente bajos frente a otros grupos nacionales y ante los nativos de origen mexicano: 5 de cada 10 cuentan con menos de 12 años de escolaridad, proporción que descendió en 5.2 puntos porcentuales (cuadro 6a).

\section{CUADRO 6A}

Indicadores de rezago social 2011 y 2016

\begin{tabular}{|c|c|c|c|c|c|c|}
\hline & \multirow[b]{2}{*}{$\begin{array}{l}\text { Estados } \\
\text { Unidos }\end{array}$} & \multicolumn{2}{|c|}{ Inmigrantes } & \multirow[b]{2}{*}{$\begin{array}{c}\text { Mexicoamericanos } \\
\text { de segunda } \\
\text { generación }\end{array}$} & \multicolumn{2}{|l|}{ Nativos } \\
\hline & & México & $\begin{array}{c}\text { No } \\
\text { mexicanos }\end{array}$ & & $\begin{array}{c}\text { Mexicoamericanos } \\
\text { de tercera } \\
\text { generación y más }\end{array}$ & $\begin{array}{c}\text { Otros } \\
\text { nativos }\end{array}$ \\
\hline \multicolumn{7}{|c|}{2011} \\
\hline $\begin{array}{l}\text { \% población en } \\
\text { condición de pobreza }\end{array}$ & 15.2 & 29.8 & 15.2 & 33.0 & 22.6 & 13.4 \\
\hline $\begin{array}{l}\text { \% población sin } \\
\text { cobertura de salud }\end{array}$ & 16.3 & 53.6 & 25.0 & 22.5 & 20.0 & 13.0 \\
\hline $\begin{array}{l}\% \text { hasta secundaria } \\
\text { sin diploma* }\end{array}$ & 17.8 & 57.5 & 18.9 & 35.4 & 28.5 & 14.5 \\
\hline \multicolumn{7}{|c|}{2016} \\
\hline $\begin{array}{l}\text { \% población en } \\
\text { condición de pobreza }\end{array}$ & 13.7 & 22.4 & 14.1 & 24.0 & 20.7 & 12.3 \\
\hline $\begin{array}{l}\text { \% población sin } \\
\text { cobertura de salud }\end{array}$ & 9.1 & 31.3 & 12.8 & 11.4 & 10.4 & 7.3 \\
\hline $\begin{array}{l}\% \text { hasta secundaria } \\
\text { sin diploma* }\end{array}$ & 16.0 & 52.3 & 17.8 & 29.2 & 24.7 & 12.8 \\
\hline
\end{tabular}

*Población de 22 años o más

Fuente: estimación de los autores con base en US Bureau of Labor Statistics, Current Population Survey (CPS), March supplement, 2016.

El trabajo precario se asocia con condiciones que se apartan de los atributos que la Organización Internacional del Trabajo (OIT) asigna al trabajo decente, como empleo seguro (bajo contrato permanente), a jornada completa, con beneficios y derechos legales. En ese sentido, el acceso a servicios de salud ofrecidos por el empleador y a un plan de pensión tiende a ser limitado para los inmigrantes (y 
en particular para los mexicanos): casi 8 de cada 10 carece de un plan de pensión y no tiene un seguro. Asimismo, la población nativa de origen mexicano ostenta indicadores de precariedad similares a los de los inmigrantes. La inseguridad laboral implica un alto riesgo de pérdida de empleo, lo que a su vez genera una serie de problemas como pobreza, desigualdad e inseguridad económica. Los inmigrantes mexicanos, junto con los nativos de origen mexicano, tienen la proporción más alta de trabajadores que laboraron únicamente una parte del año: 21.3 y 21.4 por ciento, respectivamente (cuadro 6b).

\section{CUADRO 6A}

Indicadores de precariedad laboral 2011 y 2016

\begin{tabular}{|c|c|c|c|c|c|c|}
\hline & \multirow[b]{2}{*}{$\begin{array}{l}\text { Estados } \\
\text { Unidos }\end{array}$} & \multicolumn{2}{|c|}{ Inmigrantes } & \multicolumn{3}{|c|}{ Nativos } \\
\hline & & México & $\begin{array}{c}\text { No } \\
\text { mexicanos }\end{array}$ & $\begin{array}{l}\text { Mexicoamericanos } \\
\text { de segunda } \\
\text { generación }\end{array}$ & $\begin{array}{l}\text { Mexicoamericanos } \\
\text { de tercera } \\
\text { generación y más }\end{array}$ & $\begin{array}{c}\text { Otros } \\
\text { nativos }\end{array}$ \\
\hline \multicolumn{7}{|c|}{2011} \\
\hline Tasa de desempleo & 9.5 & 11.9 & 9.2 & 14.6 & 12.7 & 9.1 \\
\hline \% laboró parte del año & 16.9 & 21.3 & 15.4 & 21.4 & 17.3 & 16.7 \\
\hline $\begin{array}{l}\% \text { sin acceso a seguro } \\
\text { médico ofrecido por el } \\
\text { empleador }\end{array}$ & 48.0 & 72.6 & 53.5 & 57.2 & 50.8 & 45.4 \\
\hline $\begin{array}{l}\% \text { sin plan de pensión } \\
\text { o de retiro ofrecido } \\
\text { por el empleador }\end{array}$ & 48.2 & 76.3 & 57.7 & 57.4 & 53.1 & 44.7 \\
\hline $\begin{array}{l}\text { Ingreso promedio } \\
\text { anual en dólares }\end{array}$ & 42481 & 25722 & 43806 & 31224 & 34036 & 43843 \\
\hline $\begin{array}{l}\text { Diferencia salarial } \\
\text { respecto a la media } \\
\text { nacional }\end{array}$ & 21 & 14 & 22 & 17 & 18 & 22 \\
\hline \multicolumn{7}{|c|}{2016} \\
\hline Tasa de desempleo & 5.3 & 5.1 & 4.3 & 7.9 & 6.9 & 5.3 \\
\hline \% laboró parte del año & 16.9 & 21.3 & 15.4 & 21.4 & 17.3 & 16.7 \\
\hline $\begin{array}{l}\text { \% sin acceso a seguro } \\
\text { médico ofrecido por el } \\
\text { empleador }\end{array}$ & 48.8 & 65.8 & 52.1 & 57.3 & 53.0 & 46.7 \\
\hline $\begin{array}{l}\% \text { sin plan de pensión } \\
\text { o de retiro ofrecido } \\
\text { por el empleador }\end{array}$ & 56.1 & 75.9 & 62.3 & 63.7 & 59.2 & 53.4 \\
\hline $\begin{array}{l}\text { Ingreso promedio } \\
\text { anual en dólares }\end{array}$ & 48047 & 31722 & 51696 & 35817 & 38600 & 49257 \\
\hline $\begin{array}{l}\text { Ingreso promedio por } \\
\text { hora en dólares }\end{array}$ & 23 & 16 & 24 & 17 & 19 & 23 \\
\hline
\end{tabular}

Fuente: estimación de los autores con base en US Bureau of Labor Statistics, Current Population Survey (CPS), March supplement, 2011 y 2016. 


\section{Indocumentados}

Un dato relevante que debe tenerse en cuenta es el número de inmigrantes indocumentados de origen mexicano, que pasó de 2 millones en 1990 (46.5 por ciento del total) a 6.5 millones en 2010 (58 por ciento). De acuerdo con estimaciones del Pew Hispanic Center (Passel y Cohn, 2016a), la cifra alcanzó su máximo histórico en 2007 y a partir de ese año descendió a 5.8 millones en 2014; aun así representa el grupo con más indocumentados en Estados Unidos. Ningún otro país cuenta con una proporción tan grande de su población en el extranjero, ubicada bajo el estigma de la irregularidad, con todo lo que ello entraña en términos de vulnerabilidad, discriminación y exclusión social. Entonces, se trata de un contrasentido y de hipocresía frente a los significativos aportes de los migrantes mexicanos a la economía estadounidense, que sólo pueden explicarse por el afán insaciable de lucro de los intereses corporativos que dominan el escenario del poder político estadounidense. La evidencia disponible sobre la contribución de la población indocumentada a la economía de Estados Unidos es contundente e irrefutable. Los datos referidos de los migrantes mexicanos en los apartados anteriores no distinguen entre la población en situación regular o irregular. Al respecto, es indispensable aclarar lo siguiente:

$\oplus$ Los inmigrantes indocumentados que viven y trabajan en Estados Unidos son consumidores y contribuyentes. Según estimaciones del Institute on Taxation and Economic Policy (Christensen, Gardner, Hill y Wiehe, 2017), los 11 millones de indocumentados pagan en impuestos estatales y locales 11 mil 740 millones de dólares (mdd), 8 por ciento de sus ingresos; lo que significa que la población mexicana indocumentada realiza una aportación importante al erario público estadounidense. Se estima que 66 por ciento de los indocumentados en 2014 tenía más de diez años de residencia y que 5.8 millones eran mexicanos (Passel y Cohn, 2016).

$\oplus$ Adicionalmente, los inmigrantes indocumentados y la mayoría de los residentes legales permanentes no tienen acceso a beneficios sociales públicos, de acuerdo con la Ley del IRCA y su posterior reforma IIRIRA (Ley IRCA, 1996).

$\oplus$ A pesar de que algunos gobiernos estatales y locales ofrecen determinados servicios públicos para los inmigrantes, el gasto estatal y local destinado específicamente para inmigrantes no autorizados representa una proporción pequeña del total, menos de 5 por ciento (CBO, 2007).

ه Más de 1.3 millones de inmigrantes de los 11 millones de indocumentados son elegibles para el programa Deferred Action for Childhood Arrivals (DACA). 
Este grupo contribuye con 2 mmdd al año en impuestos estatales y locales (8.9 por ciento de sus ingresos). La continuidad del DACA aumentaría en $425 \mathrm{mdd}$, con un incremento de 9 por ciento en la tasa impositiva (Hill y Wiehe, 2017). Entre los jóvenes elegibles para el programa, derogado por la administración de Donald Trump, 81 por ciento son inmigrantes mexicanos (DHS, 2017).

$\oplus$ La participación de los inmigrantes indocumentados en sectores económicos como construcción, agricultura, silvicultura y pesca, minería y servicios es fundamental. No suelen competir por trabajos con alta presencia de nativos y, en cambio, desempeñan un papel importante en la satisfacción de la demanda laboral y en la creación de empleos.

Lo anterior pone de relieve que la migración irregular no es ni puede ser concebida como un acto criminal; puesto que se trata de una "política de Estado» impulsada por el gobierno de Estados Unidos a través de un sistema de otorgamiento de visas que no sólo se aparta de la demanda del mercado laboral estadounidense, sino que tiene un cariz discriminatorio. No es casual el hecho de que la mitad de los indocumentados sea de origen mexicano y que 70 por ciento incorpore a los países del triángulo norte de Centroamérica: Guatemala, Honduras y El Salvador. Aunado a ello, habría que agregar que las consabidas vicisitudes, la discriminación y las violaciones sistemáticas a los derechos humanos que enfrentan los migrantes centroamericanos en tránsito por México guardan relación con esta política, solapada por el gobierno mexicano, sin el menor recato o miramiento.

\section{Transferencia de costos educativos y de reproducción social}

La inserción laboral de los migrantes en Estados Unidos comprende una transferencia de recursos derivados de los costos de reproducción educativa y social de la fuerza de trabajo. Tales costos fueron cubiertos en México a través de programas de educación, salud y asistencia social. La migración laboral representa un ahorro en formación de fuerza de trabajo para Estados Unidos, además de que el perfil demográfico de los inmigrantes corresponde a la etapa de mayor productividad. No obstante, los costos de producción y reproducción de la fuerza laboral son considerablemente menores en México.

En efecto, al tomar en cuenta el nivel de escolaridad de los migrantes mexicanos que arribaron por vez primera a Estados Unidos y el costo educativo 
implícito en la fuerza de trabajo, conforme a estimaciones del Sistema de Información sobre Migración y Desarrollo (Simde) de la Unidad Académica de Estudios del Desarrollo de la Universidad Autónoma de Zacatecas, entre 1994 y 2008 México le transfirió 83 mmdd (a precios de 2008) (Delgado, Márquez y Rodríguez, 2009). Si los niveles de escolaridad que los migrantes mexicanos llevaron consigo a Estados Unidos se hubieran alcanzado en las escuelas públicas de ese país, el costo educativo habría sido de $613 \mathrm{mmdd}$, a precios constantes de 2008, en el mismo periodo (Delgado, Márquez y Rodríguez, 2009). Las remesas canalizadas a México, que suelen ser consideradas una pérdida de recursos para Estados Unidos, habrían alcanzado apenas 30 por ciento del recurso educativo transferido por la fuerza de trabajo migrante de origen mexicano.

Estimaciones propias recientes indican que México transfirió aproximadamente 636 mmdd en 2015 por los niveles de escolaridad de los migrantes mexicanos; cabe destacar que esta estimación excluye a los inmigrantes mexicanos con estudios completos en Estados Unidos. Si se considera el costo educativo total, habría sido de $845 \mathrm{mmdd}, 209$ mmdd de los cuales corresponden a inmigrantes mexicanos con estudios en ese país. ${ }^{3}$

Además del costo educativo, la migración implica una transferencia de recursos invertidos en reproducción social, es decir, en la formación de las personas antes de emigrar. Los costos incluyen diversos rubros: bienestar público, programas sociales y gastos familiares (entre ellos una porción notable de las remesas, que contribuyen al establecimiento de una nueva fuerza laboral emigrante). Aunque los costos son difíciles de medir comprenden la base socioeconómica para la formación de capital humano. A partir de la canasta básica estimada por el Consejo Nacional para la Evaluación de la Política de Desarrollo Social, como una expresión del costo de vida, el Simde ha calculado que México transfirió 257 mmdd (a precios de 2008) a Estados Unidos entre 1994 y 2008, 1.4 veces la cantidad total de remesas recibidas durante ese periodo (Delgado, Márquez y Rodríguez, 2009). ${ }^{4}$

${ }^{3}$ Para estimar el pago de impuestos se utilizó la metodología propuesta por Héctor Rodríguez (2010) en los trabajos del Simde. Consistió en aplicar el ingreso por trabajo antes de impuestos, el porcentaje producto del impuesto federal (7.65 por ciento) y el impuesto estatal que cada individuo debe pagar. El cálculo se basa en la CPS, suplemento de marzo, y se desagrega por grupo de origen e ingresos públicos incluidos en ella, como ingresos por asistencia infantil, educativa, pública, seguridad social, desempleo, veteranos y discapacidad. Cabe mencionar que la estimación del gasto educativo entre 1994 y 2008 se sustenta también en la metodología de Rodríguez.

${ }^{4}$ A fin de calcular la transferencia de costos educativos en 2015 se utilizó la metodología propuesta por Selene Gaspar (2017) en los trabajos del Simde. Este indicador se estima mediante un procedimiento 
La sumatoria de los costos sociales y educativos (en los términos conservadores ya mencionados, de educación pública y canasta básica) arroja un total de 340 mmdd (1.8 veces la cantidad del total de remesas entre 1994 y 2008). Es muy baja la estimación porque valora los servicios proporcionados en México, en vez de hacerlo sobre costos de oportunidad de los servicios en Estados Unidos. A precios estadounidenses el valor de la transferencia educativa se eleva 7.4 veces respecto a los 83 mmdd estimados por el Simde.

El cálculo de la transferencia de la migración comienza con el inmigrante que se halla en territorio estadounidense; es decir, los costos educativos y de reproducción socioeconómica efectuados en México no se contabilizan. Si se incluyesen y las transferencias de México a Estados Unidos fuesen parte de un cálculo ajustado al valor equivalente al poder adquisitivo en dólares, sería evidente que la sociedad mexicana "subsidia» a la economía estadounidense a través de la migración laboral. Es claro que se trata de una modalidad de intercambio desigual entre ambas naciones que no suele ser reconocida y cambia radicalmente las percepciones concebidas acerca del fenómeno migratorio.

\section{Conclusiones}

Puede afirmarse que lo ya expuesto aporta diversos elementos que desmitifican la visión dominante del vínculo entre migración y desarrollo. La migración forzada está dentro del engranaje de la reestructuración capitalista bajo la égida neoliberal comandada por Estados Unidos. Los enfoques dominantes resultan limitados para dar cuenta del complejo entramado estructural que subyace a las migraciones internacionales en el capitalismo contemporáneo. Como lo devela el caso mexicano, en el contexto de la globalización neoliberal se reconfiguran las relaciones internacionales con el objeto de dar paso a una nueva división internacional del trabajo que expresa una profunda recomposición de

indirecto (proxi): los migrantes mexicanos que tienen estudios en Estados Unidos, estudios mixtos y en México (véase Gaspar, 2016) por nivel educativo con base en American Community Survey (ACS) 2015. Posteriormente se obtiene el gasto público por alumno y el nivel de escolaridad de las estadísticas que ofrece el National Center of Education Statistics U.S. La estimación del gasto público por alumno en nivel educativo se cruza con el nivel educativo de los migrantes según el lugar donde obtuvieron su formación académica, de ese modo se consigue el gasto educativo por migrante (Gaspar, 2017). Finalmente, el cálculo de la transferencia por concepto de educación toma en consideración a los que tienen estudios mixtos y en México. 
las relaciones de clase a favor del capital y, en específico, de las grandes corporaciones multinacionales. En la reconfiguración adquiere relevancia su reestructuración a fin de aprovechar los bajos costos laborales y perpetuar grandes diferenciales en la periferia del sistema y la migración laboral forzada hacia los países centrales.

Los migrantes son parte esencial del complejo entramado en virtud de que satisfacen la demanda laboral y abaratan los procesos productivos en los países centrales. En lugar de reconocer públicamente su aporte, los gobiernos de los países receptores, y en concreto el gobierno estadounidense, difunden un discurso discriminatorio y criminalizador que concibe a los inmigrantes como un peso para la sociedad receptora y un peligro a la seguridad interna. Al estigmatizar la fuerza de trabajo y en múltiples sentidos convertirla en indocumentada (mediante el establecimiento de una cuota de visas muy inferior a la demanda efectiva del mercado laboral), contribuye de manera perversa a su abaratamiento, flexibilización y vulnerabilidad. Así, el Estado desempeña una función decisiva en la regulación de los flujos migratorios de conformidad con los intereses de las clases dominantes y las corporaciones que representan. Al ocultar dicha realidad, los migrantes se muestran, ante la opinión pública de las sociedades receptoras, culpables de muchos de los males que los afligen: desmantelamiento del estado de bienestar, disminución de las clases medias, desempleo y precarización laboral, entre otros.

La exportación de fuerza de trabajo propicia modos superlativos de transferencia de excedentes que profundizan las dinámicas de desarrollo desigual. En particular, la migración laboral constituye no sólo la transferencia de los costos de formación y reproducción de la población forzada a desplazarse de sus lugares de origen, sino también la pérdida del recurso más importante para el crecimiento y desarrollo económico del país emisor, México. La creciente incorporación de fuerza de trabajo altamente calificada de la periferia al centro agrava todavía más esa problemática y compromete en extremo las posibilidades de construir un sistema nacional de innovación en el que se configuren las perspectivas de desarrollo nacional. Examinar las modalidades de intercambio desigual plantea significativos desafíos teóricos, metodológicos y empíricos para avanzar en la concepción y la caracterización del capitalismo contemporáneo y la problemática del desarrollo desigual. Los postulados que enarbolan las teorías del intercambio desigual, sustentados en las diferencias salariales que emergen de los obstáculos a la movilidad poblacional (lo que se conoce como arbitraje laboral global) abren una veta primordial analítica para el progreso de esta perspectiva. 
En síntesis: aceptar la falsa premisa de que la migración posibilita el desarrollo de los países de origen supone ignorar las causas de la migración forzada que prevalece en la actualidad, así como las múltiples contribuciones que los migrantes hacen a las economías de los países de destino, con todas las implicaciones en términos de estigmatización y criminalización de la migración laboral. Ante ese escenario y frente al pacto mundial de 2018, es imprescindible constatar que la migración, como se ha enfatizado, no representa un subsidio norte-sur, ni mucho menos una avenida para el desarrollo de los países de origen migratorio.

\section{Referencias}

Canales Cerón, Alejandro (2010), "Contribución de los migrantes latinoamericanos al PIB de los Estados Unidos de América», Sistema de Información sobre Migración y Desarrollo (Simde), en https://www.researchgate.net/publication/294180281 _Contribucion_de_los_migrantes_latinoamericanos_al_PIB_de_los_Estados_ Unidos_de_America

Canales Cerón, Alejandro (2011), «Hacia una visión comprehensiva del nexo entre migración, desarrollo y derechos humanos», Migración y Desarrollo, 9(16), p. 64.

Christensen Gee, Lisa, Matthew Gardner, Misha E. Hill y Meg Wiehe (2017), «Undocumented Immigrants' State \& Local Tax Contributions», Institute on Taxation \& Economic Policy, en https://itep.org/wp-content/uploads/immigration2017.pdf

Congress of the United States (diciembre de 2007), «The Impact of Unauthorized Immigrants on the Budgets of State and Local Governments», Congressional Budget Office Paper.

Coneval (Consejo Nacional de Evaluación de la Política de Desarrollo Social) (2008), Informe de evaluación de la política de desarrollo social en México 2008, Ciudad de México.

Cypher, James y Raúl Delgado Wise (2011), Mexico's Economic Dilemma. The Developmental Failure of Neoliberalism, New York, Rowman \& Littlefield.

Delgado Wise, Raúl y Humberto Márquez Covarrubias (2007), «Teoría y práctica de la relación dialéctica entre desarrollo y migración», Migracion y Desarrollo, 5(9), pp. $5-24$.

Delgado Wise, Raúl, Humberto Márquez Covarrubias y Héctor Rodríguez Ramírez (2009), «Seis tesis para desmitificar el nexo entre migración y desarrollo», Migración y Desarrollo (12), pp. 27-52. 
Delgado Wise, Raúl y Selene Gaspar Olvera (2012), «żQuién subsidia a quién? Contribución de los migrantes mexicanos a la economía de Estados Unidos», Observatorio del Desarrollo, 1(2), pp. 4-9.

(2017), «Demystifying irregular migration. A critical perspective», ponencia en el People's Global Action on Migration, Development and Human Rights, Berlín, en https://www.researchgate.net/publication/318216823_Demystifying_ Irregular_Migration_A_critical_perspective

Department of Homeland Security (DHS) (2017), «U.S. Citizenship and Immigration Services. Consideration of Deferred Action for Childhood Arrivals, by Fiscal Year, Quarter, Intake, Biometrics and Case Status Fiscal Year 2012-2017», Biometrics Capture Systems, Citizenship and Immigration Services Consolidated Operational Repository (CISCOR).

Gaspar Olvera, Selene (2016), iEstudiar para emigrar o emigrar para estudiar? Procesos de integración de los inmigrantes mexicanos calificados en Estados Unidos (tesis de maestría), México, Universidad Nacional Autónoma de México.

(2017), "Cambios en la dinámica laboral y contribución de los migrantes mexicanos y sus hijos a la economía de Estados Unidos» (inédito), Universidad Autónoma de Zacatecas.

Hill, Misha E. y Meg Wiehe (marzo de 2017), «State \& Local Tax Contributions of Young Undocumented immigrants», Institute on Taxation \& Economic Policy.

Illegal Immigration Reform and Immigrant Responsability Act (IIRIRA) (1996), Division C. Illegal Immigration Reform and Immigrant Responsability Act of 1996, https://epic.org/privacy/e-verify/irira-program.pdf

Mather, Mark, Linda A. Jacobsen y Kelvin M. Pollard (2015), «Aging in the United States», Population Reference Bureau. Population Bulletin, 70(2), en http://www.prb. org/pdf16/aging-us-population-bulletin.pdf

Passel, Jeffrey S. y D’Vera Cohn (2010), Unauthorized Immigrant Population: National and State Trends, Washington DC, Pew Hispanic Center, p. 31.

(20 de septiembre de 2016), "Overall Number of U.S. Unauthorized Immigrants Holds Steady Since 2009», Pew Research Center, en http://www.pewhispanic. org/2016/09/20/overall-number-of-u-s-unauthorized-immigrants-holds-steady -since-2009/\# recent-arrivals-a-smaller-share-of-u-s-unauthorized-immigrants

Organización de las Naciones Unidas (2006), Asamblea General, Migración Internacional y Desarrollo, 13 de octubre de 2016, http://www.un.org/es/comun/ docs $/$ isymbol $=\mathrm{A} / 61 / 515 \&$ Lang $=\mathrm{S}$ 
Organización de las Naciones Unidas (2013), Asamblea General, Declaración del Diálogo de Alto Nivel sobre la Migración Internacional y el Desarrollo, 1 de octubre de 2013, http://www.un.org/es/comun/docs/2̇symbol=A/68/L.5

Organización de las Naciones Unidas (2016), Asamblea General, Migración Internacionaly Desarrollo, 4 de octubre de 2016, http://www.un.org/en/development/desa/ population/migration/generalassembly/docs/A_71_296_S.pdf

Public Law 104-208-Sept. 30 (1996), «Division C-Illegal Immigration Reform and Immigrant Responsibility Act of 1996», en https://epic.org/privacy/e-verify/iiriraprogram.pdf

Robles Vázquez, Héctor (coord.) (2008), Panorama Educativo de México 2008. Indicadores del Sistema Educativo Nacional, México, Instituto Nacional para la Evaluación de la Educación (INEE), en http://www.oei.es/historico/pdf2/panorama-educativomexico-2008.pdf

Scott, Robert E. (2015), "Manufacturing job loss. Trade, not productivity, is the culprit», Economic Policy Institute. Issue Brief (402), en http://www.epi.org/files/2015/ ib402-manufacturing-job-loss.pdf

Suwandi, Intan y John Bellamy Foster (2016), «Multinational Corporations and the 30 globalization of monopoly capital: from the 1960s to the present», Monthly Review, 68(3), pp. 114-131. 